\title{
Intravital imaging of the effects of 5-fluorouracil on the murine liver microenvironment using 2-photon laser scanning microscopy
}

\author{
MASATO OKIGAMI, KOJI TANAKA, YASUHIRO INOUE, SUSUMU SAIGUSA, YOSHINAGA OKUGAWA, \\ YUJI TOIYAMA, YASUHIKO MOHRI and MASATO KUSUNOKI
}

Department of Gastrointestinal and Pediatric Surgery, Division of Reparative Medicine, Institute of Life Sciences, Mie University Graduate School of Medicine, Tsu, Mie 514-8507, Japan

Received October 12, 2014; Accepted January 18, 2016

DOI: $10.3892 / 01.2016 .4258$

\begin{abstract}
FU) is often used in the treatment of colorectal cancer. 5FU improves the median overall and disease-free survival rates and reduces recurrence rates in patients who have undergone curative surgical resection. However, in the adjuvant setting, whether 5FU eradicates clinically undetectable micrometastases in target organs such as the liver, or whether 5-FU inhibits the adhesion of circulating tumor cells has not yet been established. In the present study, $5 \mathrm{FU}$ was administered following the inoculation of red fluorescent protein-expressing HT29 cells into green fluorescent protein (GFP)-transgenic nude mice to examine its inhibitory effect. 2-photon laser scanning microscopy was performed at selected time points for time-series imaging of liver metastasis of GFP-transgenic mice. The cell number in vessels was quantified to evaluate the response of the tumor microenvironment to chemotherapy. HT29 cells were visualized in hepatic sinusoids at the single-cell level. A total of 2 hours after the injection (early stage), time-series imaging revealed that the number of caught tumor cells gradually reduced over time. In the 5FU treatment group, no significant difference was observed in the cell number in the early stage. One week after the injection (late stage), a difference in morphology was observed. The results of the present study indicated that $5 \mathrm{FU}$ eradicated clinically undetectable micrometastases in liver tissues by acting as a cytotoxic agent opposed to preventing adhesion. The present study indicated that time-series intravital 2-photon laser scanning microscopic imaging of metastatic
\end{abstract}

Correspondence to: Dr Koji Tanaka, Department of Gastrointestinal and Pediatric Surgery, Division of Reparative Medicine, Institute of Life Sciences, Mie University Graduate School of Medicine, 2-174 Edobashi, Tsu, Mie 514-8507, Japan E-mail: qouji@clin.medic.mie-u.ac.jp

Abbreviations: CRC, colorectal cancer; 5FU, 5-Fluorouracil; RFP, red fluorescent protein; GFP mouse, green fluorescent protein-transgenic mouse; TPLSM, 2-photon laser-scanning microscopy

Key words: 2-photon laser-scanning microscopy, liver metastasis, 5-fluorouracil, extravasation tumor xenografts may facilitate the screening and evaluation of novel chemotherapeutic agents with less interindividual variability.

\section{Introduction}

The incidence of colorectal cancer (CRC) has increased over time, and it has become a major health problem around the world. A previous study demonstrated that disease recurred in $25-50 \%$ of examined patients with stage II/III CRC within 5 years, despite receiving curative surgery (1). The most common site of recurrence and distant metastases is the liver, and therefore, it is important to control liver metastases to improve the prognosis of patients with CRC (2).

5-Fluorouracil (5FU) is a significant standard chemotherapy agent for advanced CRC (node positive cancer or metastatic cancer) together with leucovorin and irinotecan or oxaliplatin $(3,4)$. 5FU improves the median overall and disease-free survival rates and reduces recurrence rates in patients who have undergone curative surgical resection (5). However, in the adjuvant setting, the mechanism by which $5 \mathrm{FU}$ acts on liver metastases from CRC remains unclear. It has been hypothesized that this delayed recurrence is due to cancer dormancy. Cancer dormancy is a stage of micrometastasis but the dormant cells are clinically undetectable (6). To the best of our knowledge, no previous studies have used intravital imaging to examine the efficacy of 5FU on the microenvironment at the single-cell level in an adjuvant setting.

Since Denk et al (7) first described 2-photon laser scanning microscopy (TPLSM) in 1990, it has been used in the field of biological imaging and has become a common tool in biological laboratories. Although TPLSM has been used to observe various structures, including blood vessels, neurons, and bone marrow (8-10), little information is available on the use of TPLSM to investigate liver metastasis from CRC in vivo. Intravital TPLSM imaging has enabled the imaging of metastatic tumors, platelets in vessels, and tumor angiogenesis in addition to time-series imaging of liver metastases in the same mouse at the single-cell level (11-17). The present authors previous study established a method to visualize the real-time in vivo response to chemotherapy using intravital TPLSM, which enabled the study of dynamic interactions in living mice (11). Using this technique, the intravital imaging of the 
effects of $5 \mathrm{FU}$ on the microenvironment after tumor formation were determined (11); however, intravital imaging of micrometastasis has not previously been examined in the adjuvant setting (11). The aim of the present study was to confirm the mechanism in early stages of liver metastasis by which $5 \mathrm{FU}$ affects tumor cells at the single-cell level in living mice by using intravital TPLSM.

In the present study, the cell number in vessels in the observation field was quantified to evaluate the response to chemotherapy of the tumor microenvironment of liver metastatic xenografts. In addition, the effects of $5 \mathrm{FU}$ on the microenvironment in the adjuvant setting were studied and how $5 \mathrm{FU}$ acted on micrometastasis was observed using TPLSM.

\section{Materials and methods}

GFP nude mice and human CRC cell line. Green fluorescent protein (GFP)-expressing nude mice (C57BL/6-BALB/c-nu/nu-EGFP) were purchased from AntiCancer Japan Inc. (Osaka, Japan). GFP nude mice (8-9 weeks old) were housed in groups of $5 /$ sterile cage. The mice were kept in the animal house, according to the Institutional Animal Care Guidelines. The experimental protocols were reviewed and approved by the Animal Care and Use Committee of the Mie University Graduate School of Medicine (Tsu, Japan). A red fluorescent protein (RFP)-expressing human CRC cell line (HT29) was purchased from AntiCancer Japan Inc.

Experimental micrometastasis model. A total of 30 mice were divided into 6 groups $(\mathrm{n}=5)$. RFP-HT29 cells were injected into the spleens of GFP nude mice as a xenogenic tumor model. The tumor cells were adjusted to $1 \times 10^{7}$ cells $/ \mathrm{ml}$ as single-cell suspensions. GFP nude mice were anesthetized using isoflurane inhalation (4\%, Abbott Laboratories, Chicago, IL, USA). A total of $1 \times 10^{6}$ cells were injected into the spleens of anesthetized GFP nude mice using a 30 -gauge needle through a small incision in the left lateral abdomen.

Surgical procedures for intravital TPLSM. Immediately following injection, GFP nude mice were anesthetized via $1.0 \%$ isoflurane inhalation $(300 \mathrm{ml} / \mathrm{min})$. The liver lobe was then put into an organ-stabilizing system (Japanese Patent Application number; P2007-129723) (11).

TPLSM setup. The procedures for TPLSM setup were performed as previously described (11). Experiments were performed using an upright microscope (BX61WI; Olympus Corporation, Tokyo, Japan) and an FV1000-2P laser-scanning microscope system (Fluoview FV1000MPE, Olympus Corporation). The excitation source in TPLSM mode was Mai $\mathrm{Tai}^{\circledR} \mathrm{Ti}$ :Sapphire lasers (Spectra-Physics, Santa Clara, CA, USA), tuned and mode locked at $910 \mathrm{~nm}$. The Mai Tai laser produced light pulses of $\sim 100$ femtosecond width (repetition rate, $80 \mathrm{MHz}$ ). Laser light reached the sample through the microscope objectives, connected to an upright microscope (BX61WI). According to the depth of imaging, mean laser power at the sample was altered from 10 to $40 \mathrm{~mW}$, depending on the depth of imaging. The microscope objective lenses used in this study were as follows: UPLSAPO $4 \mathrm{x}$ (numerical aperture, 0.16), UPLSAPO 10x (numerical aperture, 0.4), and LMPLNFI/IR 60x (water dipping; numerical aperture, 0.9; working distance, $2 \mathrm{~mm}$ ) (Olympus Corporation). Data were analyzed using the FV10-ASW version 2.0 system (Olympus Corporation). TPLSM images were acquired with $512 \times 512$ pixels spatial resolution, from $210 \mu \mathrm{m}$ field of view dimension, using a pixel dwelling time of $4 \mu \mathrm{sec}$ (11).

The timing of intravital TPLSM imaging. TPLSM was performed in 3 stages of metastasis. The experimental protocol and timing of intravital TPLSM are described in Fig. 1. TPLSM imaging was performed using an upright microscope (BX61W1) and an FV1000 laser scanning microscope system (Fluoview FV1000MPE). Three stages (early, middle, and late stages) were studied in the different mice and they kept alive during the entire time. For each stage, 10 mice were used and divided into 2 groups $(\mathrm{n}=5)$. One treatment group was injected with 5FU (Sigma-Aldrich, St. Louis, MO, USA), and the other control group was injected with phosphate-buffered saline (PBS). In the early stage (Fig. 1A), TPLSM was first performed $2 \mathrm{~h}$ following the injection of RFP-HT29 cells into the GFP nude mice to observe early extravasation from circulating tumor cells. TPLSM imaging was represented clearly as a Z-stack 3-dimensional movie by scanning the images from the liver surface. Tumors were counted in a voxel of $210 \times 210 \mathrm{~nm} \times$ depth $(\sim 100 \mu \mathrm{m})$. The livers of mice in the early stage group ( 5 mice), were observed by first measuring the depth of the observation fields to quantify the cell number in the vessel in the observation fields. TPLSM was repeated at 24 and $72 \mathrm{~h}$ after the injection of RFP-HT29 cells to observe early hepatic metastasis. They were then monitored at regular intervals and randomized to receive $5 \mathrm{FU}(25 \mathrm{mg} / \mathrm{kg}$ in a $50-\mu \mathrm{l}$ volume) or an equivalent volume of PBS (50 $\mu 1)$ via intraperitoneal injection. Injections were performed 3 times a week from $1 \mathrm{~h}$ after the first injection.

In the middle stage mice (Fig. 1B), TPLSM was performed on day 4,7 , and 10 , to observe middle-stage liver metastasis. In the late stage mice (Fig. 1C), TPLSM was performed at 2, 4, and 8 weeks after the first injection of RFP-HT29 cells into the spleens of GFP nude mice to observe late-stage liver metastasis, when micrometastatic changes and macroscopic metastatic changes would have occurred.

Statistical analysis. Statistical analysis was performed using Mann-Whitney's-U-test on StatView version 5.0 software (SAS Institute, Inc., Cary, NC, USA). P $<0.05$ was considered to indicate a statistically significant difference.

\section{Results}

Early stage. The results of the TPLSM imaging were represented clearly as a Z-stack 3 -dimensional movie by scanning the images from the liver surface to a depth of $\sim 100 \mu \mathrm{m}$. On observing the livers of mice in the early stage group (5 mice), the depth of the observation fields was observed to quantify the cell number in the vessel in the observation fields, and the depth of $\sim 100 \mu \mathrm{m}$ was observed multiple times (Fig. 2A). The cells were counted in a voxel of $210 \times 210 \mathrm{~nm} \times$ depth $(\sim 100 \mu \mathrm{m})$. The number of tumor cells caught in the hepatic sinusoid was counted and the mean was calculated for 
A

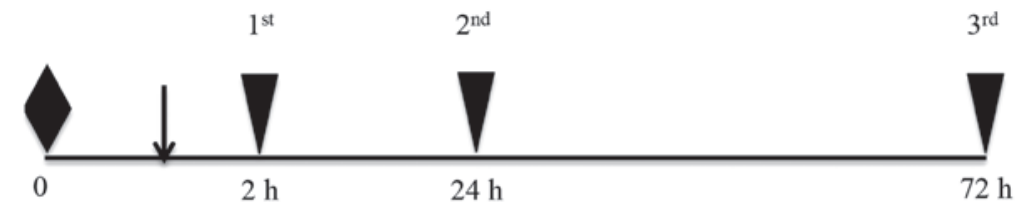

B
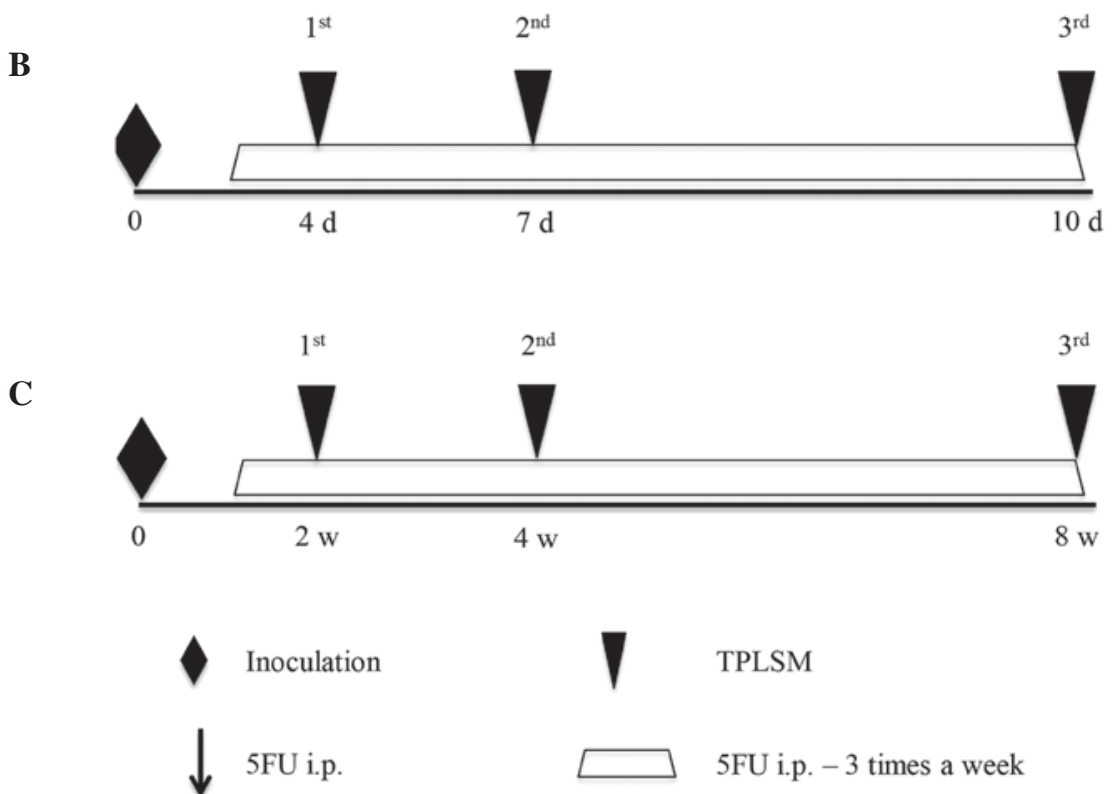

Figure 1. Experimental protocol and timing of intravital TPLSM. (A) TPLSM was performed 2, 24, and $72 \mathrm{~h}$ after the injection of cells in the same mouse to observe the early stages of colorectal liver metastasis. (B) TPLSM was performed 4, 7, and 10 days after the injection of cells in the same mouse to observe the middle stages of colorectal liver metastasis. (C) TPLSM was performed 2, 4, and 8 weeks after the injection of cells in the same mouse to observe the late stages of colorectal liver metastasis. 5FU, 5-Fluorouracil; TPLSM, 2-photon laser-scanning microscopy.

A

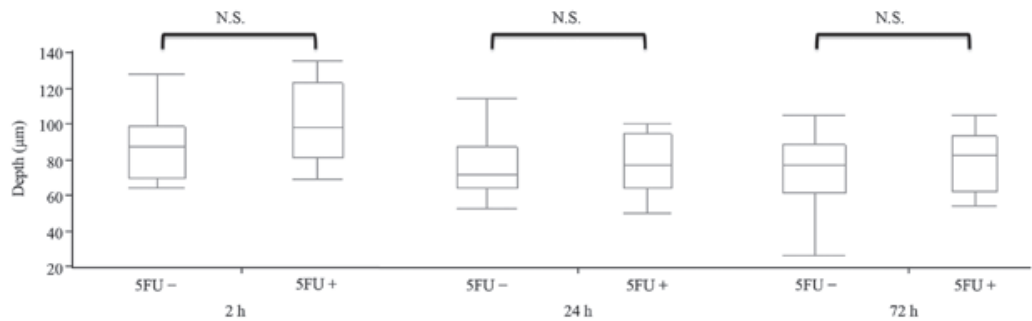

B

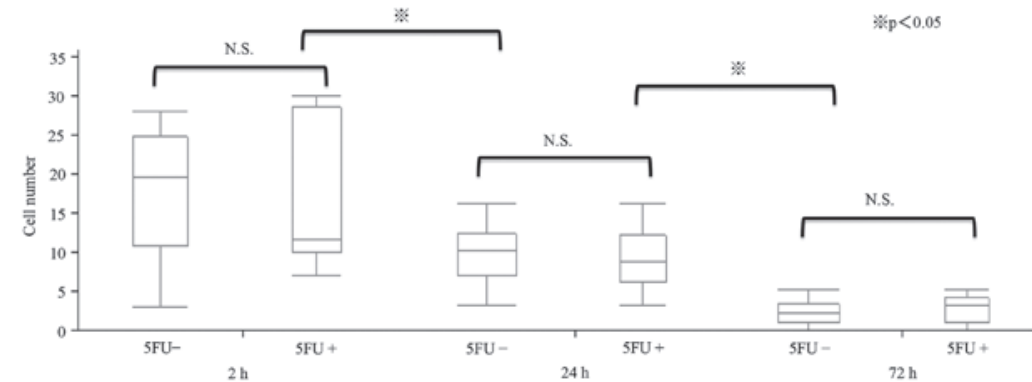

Figure 2. Quantification of cells in the vessel after inoculation (2, 24, and $72 \mathrm{~h})$ in the same mouse. The mean number of cells was calculated for 3 mice. (A) Tumors were counted in a voxel of 210x210 nm x depth $(\mu \mathrm{m})$. The depth of the observation field was not significantly different among the livers. (B) In each Voxel, the cell number was significantly different at 2, 24, and 72 h. 5FU, 5-Fluorouracil.

5 mice. The depth observed was not significantly different among the different fields of the liver. The number of cells in the hepatic sinusoid gradually reduced over time $(\mathrm{P}<0.05$;
Fig. 2B). At 2 h, 17.9 \pm 7.9 cells were counted in the voxel. At 24 and $72 \mathrm{~h}, 9.5 \pm 3.7$ and $2.3 \pm 0.4$ cells were counted in the voxel, respectively. 


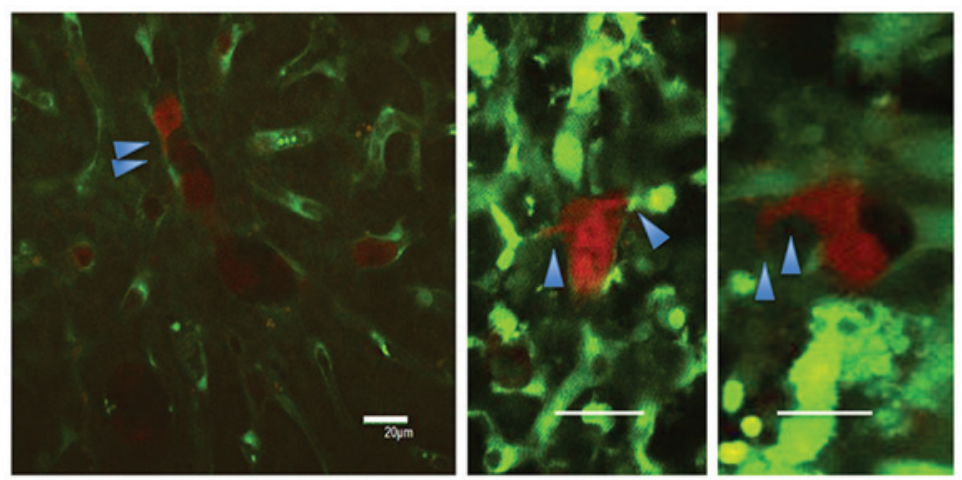

Figure 3. Macroscopic findings of invadopodia (scale bar, $20 \mu \mathrm{m}$ ).

Regarding the early stage in vivo response to 5FU, similar results were observed for the 5FU-treated groups at the 3 time points $(2,24$, and $72 \mathrm{~h})$. The tumor cell count at $2 \mathrm{~h}$ was $16.5 \pm 9.2$ in the $5 \mathrm{FU}$ group, compared with $17.9 \pm 7.9$ in the control group. At 24 and 72 h, 8.8 \pm 3.9 and $2.2 \pm 1.8$ cells were counted in the voxel, respectively, in the 5FU-treated groups.

Regarding the 2-h intravital TPLSM, several RFP-HT29 cells were present with platelets in the hepatic sinusoids, and the majority of these cells were circle-shaped. In the 24-h intravital TPLSM, a number of RFP-HT29 cells had been removed from the sinusoids and the number of adhesive platelets had increased. In addition, invadopodia were observed in both groups (Fig. 3). A greater number of invadopodia were observed in the control groups compared with the 5FU groups, however the difference in number was not significant. For the 24-h TPLSM, extravascular metastatic foci of RFP-HT29 cells were observed and a small number of cells were present in the hepatic sinusoids. Due to the presence of inflammation, it was not clear whether the tumor cells within the surrounding hepatic tissues had changed. These changes occurred under the influence of inflammation. Similar results were observed for the 5FU groups (Fig. 4A).

Middle and late stages. Certain differences in microscopic appearance were observed 10 days after the injection of RFP-HT29 cells. In the control group, metastatic foci were observed, but in the 5FU treated group, cell fragments due to apoptosis were observed (Fig. 4B).

Two weeks after the injection of RFP-HT29 cells, additional differences were noted in the macroscopic appearance compared with the appearance at 10 days. At the 2 -week intravital TPLSM, cell apoptosis was observed. At the 4-week TPLSM, apparent differences were noted between the 5FU and control groups. The number of extravasated HT29 cells was reduced in the 5FU treatment group, and an increase in cell fragments was observed. An increase in the number of metastatic foci of HT29 cells was observed at the 4-week TPLSM compared with at the 2-week TPLSM. At the 8-week TPLSM, increased liver metastatic colonization and tumor vessels were visualized in the control groups; in the 5FU-treated groups, a markedly reduced number of HT29 cells were observed in the voxel (Fig. 4C). In addition, liver metastases were identified as macroscopic findings (Fig. 5). The results indicate that 5FU inhibited the growth of CRC tumors in vivo.

\section{Discussion}

The findings of the present study revealed a correlation between the inhibition of recurrence by $5 \mathrm{FU}$ and the mechanism by which $5 \mathrm{FU}$ acts on liver metastases from CRC, at the single-cell level. For stage II/III CRC, surgical removal of the primary tumor is the only available curative intervention; however, $25-50 \%$ of patients relapse within 5 years (1). Relapse may be due to undetectable tumor cells remaining following curative surgery. After curative surgery, the need for effective chemotherapy to inhibit recurrence is clear. In the 1990s, 2 clinical trials revealed that 5FU-based regimens improved overall or recurrence-free survival in the adjuvant setting $(5,18)$. The aim of adjuvant chemotherapy is to reduce the risk of local and distal recurrence of CRC.

5FU, a widely used cytotoxic agent, is a pyrimidine analog that is converted intracellularly to a number of products that bind to the enzyme thymidylate synthase and interfere with DNA replication. At present, previous studies have investigated the efficacy of 5FU in vitro, and a number of studies have examined the effects of $5 \mathrm{FU}$ in subcutaneous xenografts preclinically $(19,20)$. However, to the best of our knowledge, no previous study has evaluated the effect of 5FU on micrometastatic foci in the process of suppressing liver metastasis following surgery in vivo. Previous studies have reported differences in the effects of treatment on subcutaneous and liver metastatic xenografts $(21,22)$. Therefore, the present study used a liver metastatic xenograft as a preclinical model to evaluate an anticancer drug. In the present authors previous study, a method for intravital TPLSM imaging of liver metastasis was established at the single-cell level using GFP-expressing mice $(15,16)$. In addition, the authors reported that TPLSM enabled observation at 3 time points and that the response of metastatic tumor cells to 5FU in the microenvironment could be visualized (11). Martin et al (23) reported the ex vivo examination of individual tumor cells in the liver and micrometastasis in situ by using 2-photon microscopy, and they also reported the influence of different microenvironments on tumor cell extravasation. However, no prior study has investigated the interaction of tumor cells with their microenvironment at the earliest steps of metastasis in vivo. In the present study, the response of circulating tumor cells to $5 \mathrm{FU}$ at the single-cell level in the 3-dimensional microenvironment was visualized using $\mathrm{Z}$-stack imaging in the early stages of liver metastasis. To the best of our knowledge, this 
A

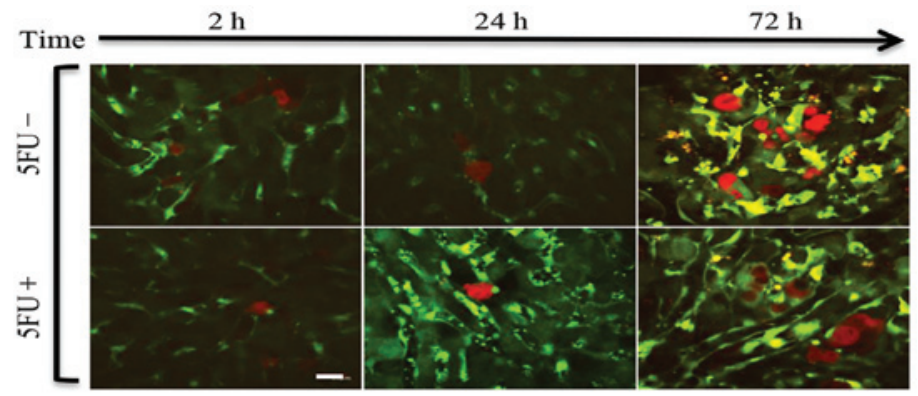

$\mathbf{B}$

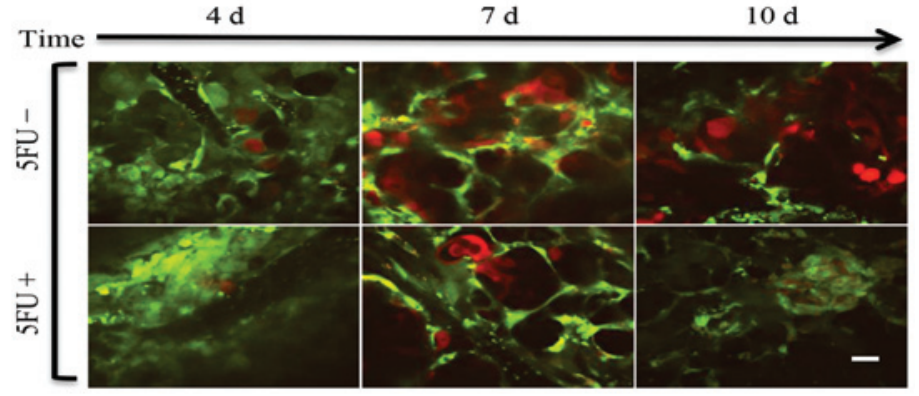

C

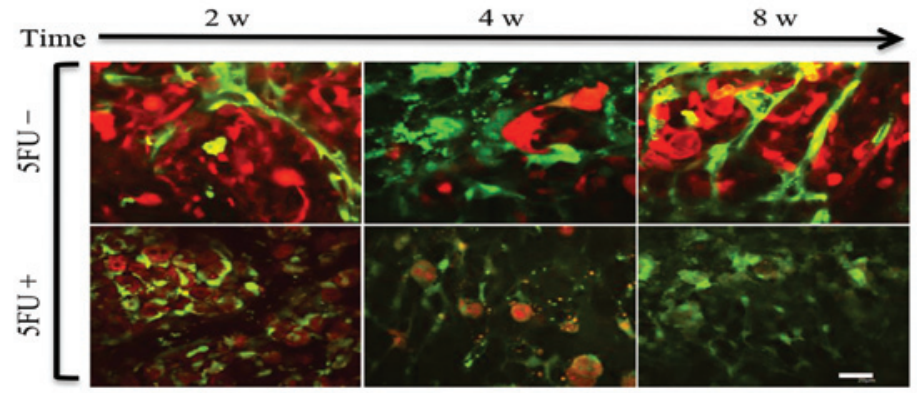

Figure 4. (A) Early-stage TPLSM images of the liver in each group. Representative images were taken in the same mouse. Arrested tumor cells in the hepatic sinusoid gradually reduced over time. Images were captured 2, 24, and $72 \mathrm{~h}$ after the injection of tumor cells in 5FU-treated and untreated mice (scale bar, $20 \mu \mathrm{m}$ ). (B) Middle stage of time course TPLSM images of the liver in each group. Representative images were captured in the same mouse, 4, 7 and 10 days after injection with or without 5FU treatment. Cell fragments due to apoptosis were observed (scale bar, $20 \mu \mathrm{m}$ ). (C) Late-stage TPLSM images of the liver in each group. Representative images were captured in the same mouse, 2, 4, and $8 \mathrm{w}$ after the injection of tumor cells in 5FU-treated and untreated mice. Extravasated tumor cells in the hepatic tissues (scale bar, $20 \mu \mathrm{m}$ ).

A

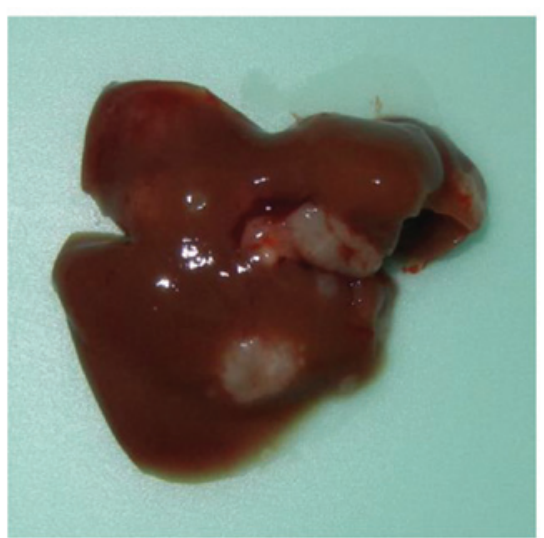

B

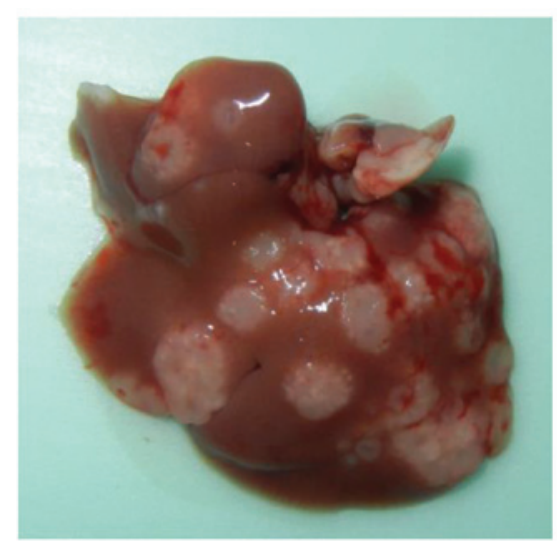

Figure 5. Macroscopic findings of liver metastasis. (A) 5FU treated, (B) control.

is the first demonstration of the effect of 5FU on liver micrometastasis at the single-cell level prior to the development of liver metastasis in the murine model at the earliest steps of metastasis. The point at which 5FU affects the metastatic process was determined. In the early stage, there was little change in the number of metastatic foci in response to 5FU.
Morphological changes such as invadopodia and apoptosis were also visualized.

The results of the present study revealed that 5FU did not prevent tumor adhesion to the liver sinusoids and supported the arguments of Warusavitarne et al (24) that 5FU treatment does not influence invasion and metastasis in microsatellite-unstable 
colorectal cancer in vitro. The results of the present study also demonstrated that 5FU acted on HT29 cells as a cytotoxic agent after the cells extravasated into the tissue parenchyma. These results may lead to a better understanding of the earliest steps of tumor-host interactions in the liver microenvironment.

In the middle and late stage groups, the mechanism by which 5FU acted on the extravasated cells and the tumor cells that began to proliferate were observed. As expected, $5 \mathrm{FU}$ inhibited the growth of tumors. The present authors previously reported that cancer cells in metastatic foci were involved in peripheral tumor cell fragmentation and central tumor necrosis in the body and that platelet aggregation occurred on endothelial cells within the metastatic-focus tumor vessel (11). This observation indicated that the tumor vessel endothelial cell is an obstacle to successfully treating metastasis. A number of phenomena, including peripheral tumor cell fragmentation and central tumor necrosis, are observed when 5-FU is prescribed to patients by metronomic scheduling. The findings of the present study supported this hypothesis at the single-cell level by using a liver metastatic mouse model under real-time TPLSM. As Kerbel et al (25) and Colleoni et al (26) proposed, the endothelial damage or coagulation abnormality or tumor vascular disorder may be associated with the mechanisms of metronomic chemotherapy.

In metastatic cancer, the extravasation and proliferation of cancer cells in the target organ and the interactions between cancer cells and target organ stromal cells serve important roles. To determine the effect of novel anticancer agents for the purpose of metastatic control, it is important to evaluate their therapeutic effects on cancer cells and host cells in the microenvironment of metastatic foci in a liver metastasis model. Therefore, the tumor-host interaction needs to be observed at the single-cell level.

TPLSM has 2 major functions: i) The observation of living tissues and cells (low toxicity) and ii) deep observation (high permeability). TPLSM is attracting attention as a tool to visualize the target and anticancer mechanism of novel cancer drugs. TPLSM has been used to investigate bone marrow, blood vessels, and the thymus (13,27-29). Intravital TPLSM may reveal information missed by conventional histopathological and molecular biological analyses. TPLSM may be used to monitor the spatiotemporal relationships between cancer cells and endothelial or blood cells.

Adjuvant chemotherapy often consists of cytotoxic drugs, and is commonly used to target CRC. However, adjuvant chemotherapy does not only target CRC cells. For example, the target of bevacizumab is the host tissue (blood vessels).

A previous study demonstrated whole-body imaging in metastatic models (30). Compared with whole-body imaging, the strengths of TPLSM include the ability to visualize tumors clearly at the single-cell level to evaluate drug efficacy.

The present authors previously reported that TPSLM enables the observation of tumor vessels in the surrounding stroma in the same mouse at different time points (11). In the present study, the effects of a cytotoxic drug at different time points were observed at the cellular level. This strategy may be applied to observe the efficacy of host-targeted drugs such as Bevacizumab by using the methodology of quantification.

A limitation of the present study is that intravital TPLSM only captures images to a depth of $200 \mu \mathrm{m}$ in liver tissues, and thus, it is not easily applicable to human tissues. In addition, the organ-stabilizing system minimizes the artifacts caused by heart and respiratory movements, but it is a technically challenging procedure. There is therefore a need to improve the procedure. However, technological advancements in fluorescence microscopy will aid in revealing the mechanisms of cancer progression and provide visual targets for novel therapeutics.

In conclusion, in the field of biological imaging, intravital TPLSM may be a powerful tool to reveal the mechanisms of the effects of drugs on cancer progression at the single-cell level and to enable the visualization of targets of novel anticancer therapeutics.

\section{Acknowledgements}

The present study was supported by grants from the Ministry of Education, Culture, Sports, Science, and Technology of Japan (KAKENHI; grant nos., 22591484, 21591723 and 21390377).

\section{References}

1. Desch CE, Benson AB III, Somerfield MR, Flynn PJ, Krause C, Loprinzi CL, Minsky BD, Pfister DG, Virgo KS and Petrelli NJ; American Society of Clinical Oncology: Colorectal cancer surveillance: 2005 update of an American society of clinical oncology practice guideline. J Clin Oncol 23: 8512-8519, 2005.

2. Manfredi S, Lepage C, Hatem C, Coatmeur O, Faivre J and Bouvier AM: Epidemiology and management of liver metastases from colorectal cancer. Ann Surg 244:254-259, 2006.

3. No authors listed: Chemotherapy of metastatic colorectal cancer: Fluorouracil plus folinic acid and irinotecan or oxaliplatin. Prescrire Int 14:230-233, 2005.

4. Kong L, Wang X, Zhang K, Yuan W, Yang Q, Fan J, Wang P and Liu Q: Gypenosides synergistically enhances the anti-tumor effect of 5-fluorouracil on colorectal cancer in vitro and in vivo: A role for oxidative stress-mediated DNA damage and p53 activation. PLoS One 10: e0137888, 2015.

5. Moertel CG, Fleming TR, Macdonald JS, Haller DG, Laurie JA, Goodman PJ, Ungerleider JS, Emerson WA, Tormey DC and Glick JH: Levamisole and fluorouracil for adjuvant therapy of resected colon carcinoma. N Engl J Med 322: 352-358, 1990.

6. Wikman H, Vessella R and Pantel K.: Cancer micrometastasis and tumour dormancy. APMIS 116: 754-770, 2008.

7. Denk W, Strickler JH and Webb WW: Two-photon laser scanning fluorescence microscopy. Science 248: 73-76, 1990.

8. Hickey MJ and Kubes P: Intravascular immunity: The host-pathogen encounter in blood vessels. Nat Rev Immunol 9: 364-375, 2009.

9. Grutzendler J, Yang G, Pan F, Parkhurst CN and Gan WB: Transcranial two-photon imaging of the living mouse brain. Cold Spring Harb Protoc 2011: pdb.prot065474, 2011.

10. Sumen C, Mempel TR, Mazo IB and von Andrian UH: Intravital microscopy: Visualizing immunity in context. Immunity 21 : 315-329, 2004.

11. Tanaka K, Okigami M, Toiyama Y, Morimoto Y, Matsushita K, Kawamura M, Hashimoto K, Saigusa S, Okugawa Y, Inoue $\mathrm{Y}$, et al: In vivo real-time imaging of chemotherapy response on the liver metastatic tumor microenvironment using multiphoton microscopy. Oncol Rep 28: 1822-1830, 2012.

12. Toiyama Y, Mizoguchi A, Okugawa Y, Koike Y, Morimoto Y, Araki T, Uchida K, Tanaka K, Nakashima H, Hibi M, et al: Intravital imaging of DSS-induced cecal mucosal damage in GFP-transgenic mice using two-photon microscopy. J Gastroenterol 45: 544-553, 2010.

13. Koike Y, Tanaka K, Okugawa Y, Morimoto Y, Toiyama Y, Uchida K, Miki C, Mizoguchi A and Kusunoki M: In vivo real-time two-photon microscopic imaging of platelet aggregation induced by selective laser irradiation to the endothelium created in the beta-actin-green fluorescent protein transgenic mice. J Thromb Thrombolysis 32: 138-145, 2011. 
14. Morimoto Y, Tanaka K, Toiyama Y, Inoue Y, Araki T, Uchida K, Kimura K, Mizoguchi A and Kusunoki M: Intravital three-dimensional dynamic pathology of experi- mental colitis in living mice using two-photon laser scanning microscopy. J Gastrointest Surg 15: 1842-1850, 2011.

15. Tanaka K, Morimoto Y, Toiyama Y, Okugawa Y, Inoue Y, Uchida K, Kimura K, Mizoguchi A and Kusunoki M: Intravital dual-colored visualization of colorectal liver metastasis in living mice using two photon laser scanning microscopy. Microsc Res Tech 75: 307-315, 2012.

16. Tanaka K, Morimoto Y, Toiyama Y, Matsushita K, Kawamura M, Koike Y, Okugawa Y, Inoue Y, Uchida K, Araki T, et al: In vivo time-course imaging of tumor angiogenesis in colorectal liver metastases in the same living mice using two-photon laser scanning microscopy. J Oncol 2012: 265487, 2012

17. Tanaka K, Toiyama Y, Inoue Y, Uchida K, Araki T, Mohri Y, Mizoguchi A and Kusunoki M: Intravital imaging of gastrointestinal diseases in preclinical models using two-photon laser scanning microscopy. Surg Today 43: 123-129, 2013.

18. De Gramont A, Krulik M, Cady J, Lagadec B, Maisani JE, Loiseau JP, Grange JD, Gonzalez-Canali G, Demuynck B and Louvet C: High-dose folinic acid and 5-fluorouracil bolus and continuous infusion in advanced colorectal cancer. Eur J Cancer Clin Oncol 24: 1499-1503, 1988.

19. Torimura T, Ueno T, Taniguchi E, Masuda H, Iwamoto $H$, Nakamura T, Inoue K, Hashimoto O, Abe M, Koga H, et al: Interaction of endothelial progenitor cells expressing cytosine deaminase in tumor tissues and 5-fluorocytosine administration suppresses growth of 5-fluorouracil-sensitive liver cancer in mice. Cancer Sci 103: 542-548, 2012.

20. Hoffmann D, Bayer W and Wildner O: In situ tumor vaccination with adenovirus vectors encoding measles virus fusogenic membrane proteins and cytokines. World J Gastroenterol 13 : 3063-3070, 2007.

21. Wilmanns C, Fan D, Obrian C, Radinsky R, Bucana C, Tsan R and Fidler I: Modulation of doxorubicin sensitivity and level of P-glycoprotein expression in human colon-carcinoma cells by ectopic and orthotopic environments in nude-mice. Int J Oncol 3: 413-422, 1933
22. Wilmanns C, Fan D, O'Brian CA, Bucana CD and Fidler IJ: Orthotopic and ectopic organ environments differentially influence the sensitivity of murine colon carcinoma cells to doxorubicin and 5-fluorouracil. Int J Cancer 52: 98-104, 1992.

23. Martin MD, Kremers GJ, Short KW, Rocheleau JV, Xu L, Piston DW, Matrisian LM and Gorden DL: Rapid extravasation and establishment of breast cancer micrometastases in the liver microenvironment. Mol Cancer Res 8: 1319-1327, 2010.

24. Warusavitarne J, Ramanathan P, Kaufman A, Robinson BG and Schnitzler M: 5-fluorouracil (5FU) treatment does not influence invasion and metastasis in microsatellite unstable (M SI-H) colorectal cancer. Int J Colorectal Dis 21: 625-631, 2006.

25. Kerbel RS and Kamen BA: The anti-angiogenic basis of metronomic chemotherapy. Nat Rev Cancer 4: 423-436, 2004.

26. Colleoni M, Orlando L, Sanna G, Rocca A, Maisonneuve P, Peruzzotti G, Ghisini R, Sandri MT, Zorzino L, Nolè F, et al: Metronomic low-dose oral cyclophosphamide and methotrexate plus or minus thalidomide in metastatic breast cancer: Antitumour activity and biological effects. Ann Oncol 17: 232-238, 2006.

27. Ishii M, Egen JG, Klauschen F, Meier-Schellersheim M, Saeki Y, Vacher J, Proia RL and Germain RN: Sphingosine-1-phosphate mobilizes osteoclast precursors and regulates bone homeostasis. Nature 458: 524-528, 2009

28. Hickey MJ and Kubes P: Intravascular immunity: The host-pathogen encounter in blood vessels. Nat Rev Immunol 9: 364-375, 2009.

29. Bousso P, Bhakta NR, Lewis RS and Robey E: Dynamics of thymocyte-stromal cell interactions visualized by two-photon microscopy. Science 296: 1876-1880, 2002.

30. Yang M, Baranov E, Jiang P, Sun FX, Li XM, Li L, Hasegawa S, Bouvet M, Al-Tuwaijri M, Chishima T, et al: Whole-body optical imaging of green fluorescent protein-expressing tumors and metastases. Proc Natl Acad Sci USA 97: 1206-1211, 2000. 